\title{
TIA: Vitamin-K-Antagonisten versus Thrombozytenaggregationshemmer
}

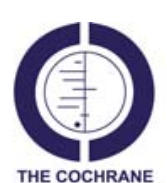

THE COCHRANE
Nach einer transitorisch ischämischen Attacke (TIA) bzw. einem nicht-invalidisierenden Schlaganfall embolischer

Genese besteht eine klare Indikation zur oralen Antikoagulation mit einem Vitamin-K-Antagonisten. Ein aktuelles Cochrane-Review beschäftigt sich mit der Frage, ob die orale Antikoagulation auch bei kleineren zerebralen Ereignissen arterieller Genese sinnvoll ist.

Einleitung: Das jährliche Risiko, nach einer TIA an einem großen zerebralen Insult oder einem Myokardinfarkt zu sterben, liegt zwischen $4 \%$ und $11 \%$. Für Myokardinfarkte war in der Vergangenheit gezeigt worden, dass die orale Antikoagulation der Thrombozytenaggregationshemmung bei der Verhinderung schwerwiegender vaskulärer Ereignisse überlegen ist.

Studien: Für das Cochrane-Review wurden 8 Studien mit insgesamt 5762 Patienten ausgewählt, welche die Sekundärprävention nach nicht-embolisch bedingter TIA bzw. kleinerem zerebralem Insult untersuchten. Es wurden entweder VitaminK-Antagonisten oder Medikamente zur Thrombozytenaggregation eingesetzt. Alle Studien wiesen ein kontrolliertes, randomisiertes Design auf. Das mittlere Alter der Patienten lag in den eingeschlossenen Studien zwischen 60 und 68 Jahren. Die Intensität der Antikoagulation mit Vitamin-K-Antagonisten wurde anhand der INR-Werte (International Normalized Ratio) in niedrig, mittel und hoch eingeteilt. Als Endpunkte definierten die Autoren die Gesamtmortalität sowie vaskuläre Ereignisse wie Schlaganfall und Myokardinfarkt und deren Folgen. Außerdem wurde das Auftreten von Blutungskomplikationen unter der jeweiligen Therapie erfasst.

Ergebnisse: Die Auswertungen zeigten, dass die orale Antikoagulation mit Vitamin-K-Antagonisten in keiner Dosierung der Thrombozytenaggregationshemmung bei der Verhinderung vaskulärer Ereignisse überlegen war. In der Gruppe der mit mittlerer Intensität oral antikoagu-

Herausgeber:

Dr. med. Jörg Meerpoh

Prof. Dr. rer. nat. Gerd Antes lierten Patienten (INR 2,0-3,6) lag das relative Risiko (RR) bei 0,80 (95\%-Konfidenzintervall [KI] 0,56-1,14), bei der oralen Antikoagulation mit hoher Intensität (INR 3,0-4,5) wurde ein RR von 1,02 (95\%-KI 0,49-2,13) verzeichnet. Das Blutungsrisiko war dagegen bei INR-Werten zwischen 2,0 und 4,5 signifikant erhöht (mittlere Intensität der oralen Antikoagulation: RR 1,93; 95\%-KI 1,27-2,94; hohe Intensität: RR 9,0; 95\%-KI 3,9-21).

\section{Fazit der Cochrane-Autoren}

Nach einer nicht-embolisch bedingten TIA oder einem kleineren zerebralen Insult sind Vitamin-K-Antagonisten der Thrombozytenaggregationshemmung in der Sekundärprophylaxe nicht überlegen. Die orale Antikoagulation mit Vitamin-K-Antagonisten führt aber, sofern INR-Werte zwischen 2,0 und 4,5 erreicht werden, im Vergleich zur Thrombozytenaggregationshemmung zu einem signifikant erhöhten Blutungsrisiko. Die Autoren weisen aber darauf hin, dass möglicherweise die mit einem geringeren Blutungsrisiko vergesellschafteten neuen oralen Antikoagulanzien (direkte Thrombin- und Faktor Xa-Inhibitoren) in zukünftigen Studien mit der Thrombozytenaggregationshemmung verglichen werden sollten.

\section{Dr. med. Katharina Franke, Darmstadt}

Originalarbeit: De Schryver ELLM et al. Vitamin K antagonists versus antiplatelet therapy after transient ischaemic attack or minor ischaemic stroke of presumed arterial origin. Cochrane Database of Systematic Reviews 2012, Issue 9

DOI: 10.1002/14651858.CD001342.pub3 www.thecochranelibrary.com
Die Hauptursache einer transitorisch ischämische Attacke (TIA) oder eines ischämischen Schlaganfalls sind intrazerebrale Gefäßverschlüsse kardioembolischer (meist bei Vorhofflimmern) oder atherosklerotischer Genese. Da das Rezidivrisiko bei beiden Ursachen hoch ist, und zudem Patienten nach zerebraler Ischämie auch ein erhöhtes gesamtes kardiovaskuläres Risiko haben, hat die Sekundärprävention einen hohen Stellenwert. Neben einer optimalen Therapie der kardiovaskulären Risikofaktoren hat die antithrombotische Therapie eine große Bedeutung. Letztere muss differenzialtherapeutisch in Abhängigkeit von der Genese des zerebrovaskulären Ereignisses (kardioembolisch vs. atherosklerotisch) eingesetzt werden.

Aktuell wurden die Empfehlungen zur Sekundärprophylaxe des ischämischen Insults in einer deutschsprachigen S3Leitlinie zusammengefasst (siehe QRCode unten): Patienten mit Vorhofflimmern nach zerebrovaskulärem Ereignis profitieren in hohem Maße von einer oralen Antikoagulation mit Vitamin-KAntagonisten oder den neuen direkten oralen Antikoagulanzien. Thrombozytenfunktionshemmer sind in dieser Indikation nicht effektiver als Placebo, aber mit einem höheren Blutungsrisiko assoziiert und haben in dieser Indikation keinen Stellenwert. Bei zerebrovaskulärem Ereignis atherosklerotischer Genese dagegen ist ASS effektiv und reduziert sowohl die zerebrovaskuläre als auch die gesamte kardiovaskuläre Ereignisrate und die gesamte Mortalität.

Die vorgestellte Cochrane-Analyse unterstützt die aktuellen Leitlinienempfehlungen.

\section{PD Dr. J. Beyer-Westendorf}

\section{Prof. Dr. N. Weiss}

UniversitätsGefäßCentrum und Medizinische Klinik III - Bereich Angiologie

Universitätsklinikum Carl Gustav Carus an der Technischen Universität Dresden

Interessenkonflikte: Die Autoren erklären, Forschungsförderung, Vortrags- und Beraterhonorare der Firmen Boehringer Ingelheim (Dabigatran), Bayer Vital (Rivaroxaban) und Bristol-Meyers Squibb/Pfizer Pharma (Apixaban) erhalten zu haben.

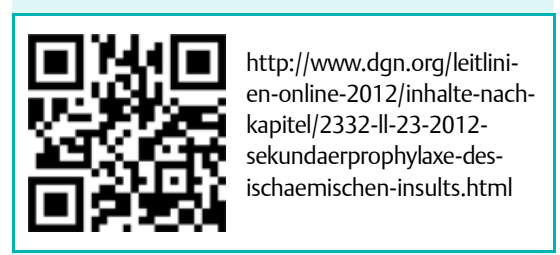

